\title{
$k$-Colour partitions of acyclic tournaments
}

\author{
Paulo Barcia \\ Universidade Nova de Lisboa, Faculdade de Economia \\ Campus de Campolide, 1099-032 Lisboa, Portugal \\ barcia@fe.unl.pt \\ J. Orestes Cerdeira* \\ Instituto Superior de Agronomia, Univ. Técnica de Lisboa \\ Tapada da Ajuda, 1349-017 Lisboa, Portugal \\ orestes@isa.utl.pt
}

Submitted: Jun 27, 2002; Accepted: Aug 21, 2004; Published: Jan 7, 2005

Mathematics Subject Classifications: 05C20, 52B05

\begin{abstract}
Let $G_{1}$ be the acyclic tournament with the topological sort $0<1<2<\cdots<$ $n<n+1$ defined on node set $N \cup\{0, n+1\}$, where $N=\{1,2, \ldots, n\}$. For integer $k \geq 2$, let $G_{k}$ be the graph obtained by taking $k$ copies of every arc in $G_{1}$ and colouring every copy with one of $k$ different colours. A $k$-colour partition of $N$ is a set of $k$ paths from 0 to $n+1$ such that all arcs of each path have the same colour, different paths have different colours, and every node of $N$ is included in exactly one path. If there are costs associated with the arcs of $G_{k}$, the cost of a $k$-colour partition is the sum of the costs of its arcs. For determining minimum cost $k$-colour partitions we describe an $O\left(k^{2} n^{2 k}\right)$ algorithm, and show this is an NP-hard problem. We also study the convex hull of the incidence vectors of $k$-colour partitions. We derive the dimension, and establish a minimal equality set. For $k>2$ we identify a class of facet inducing inequalities. For $k=2$ we show that these inequalities turn out to be equations, and that no other facet defining inequalities exists besides the trivial nonnegativity constraints.
\end{abstract}

\section{Introduction}

Let $G_{1}$ be the acyclic tournament with the topological sort $0<1<2<\cdots<n<n+1$ defined on node set $N \cup\{0, n+1\}$, where $N=\{1,2, \ldots, n\}$. The arcs of $G_{1}$ are therefore the pairs $(i, j)$, with $i<j$. Given an integer $k \geq 2$, consider the (di)graph $G_{k}$ obtained by

*This author's research was financially supported by the Portuguese Foundation for Science and Technology (FCT) 
taking $k$ copies of every arc in $G_{1}$ and colouring every copy with one of $k$ different colours. A $k$-colour partition of $N$ ( $k$-CP, for short) is a set of $k$ paths from 0 to $n+1$ such that all arcs of each path have the same colour, different paths have different colours, and every node of $N$ is included in exactly one path.

The $k$-CPs are related with path partitions of digraphs [2]. A $k$ path partition (also called $k$-path factor) of a digraph $D$ is a set of $k$ pairwise node disjoint paths spanning $D$. The path covering number of $D$ is the minimum integer $k>0$ for which $D$ has a $k$ path partition. The path covering number of an acyclic digraph can be easily determined by means of network flows, see chapter 5 of [1], where some applications of path partitions for acyclic digraphs are also discussed. The $k$-CPs generalize path partitions for acyclic graphs having different colour arcs. $k$-CPs are $k$ path partitions in which all arcs of each path are required to have the same colour. Some questions regarding graphs with different colour arcs are addressed in chapter 11 of [1]. However, there is no direct relation between those questions and $k$-CPs.

If each arc of $G_{k}$ has a real cost, the cost of a $k$-CP is the sum of the costs of the arcs of its $k$ paths. Minimum cost $k$-CPs may be found by means of shortest path techniques. In section 2 we describe an $O\left(k^{2} n^{2 k}\right)$ algorithm, and show that determining minimum cost $k$-CPs is NP-hard. Minimum cost $k$-CPs can be used to model scheduling problems in which $k$ different machines are to be assigned to process $n$ jobs having precedence requirements. Hence, $k$-CPs generalize $k$ path partitions on acyclic digraphs, for the case of nonidentical machines.

The knowledge of the polytope associated with a combinatorial optimization problem allows the use of linear programming techniques to solve the problem. Even when only some of its facets are known, that information often helps to design successful branch and cut algorithms. (See for example [5] for the use of polyhedra in the context of combinatorial optimization.)

We study the convex hull of the incidence vectors (in $\mathbb{R}^{k(n+2)(n+1) / 2}$ ) of $k$-CPs. In section 3 we consider the case $k=2$. We give the dimension of the polytope, a complete minimal equality set, and we show that no other facet exists besides the trivial nonnegativity constraints. A minimal equality set for $k>2$ is obtained generalizing some of the equations described for $k=2$. All other equations generalize as valid inequalities, each defining a facet of the convex hull of the incidence vectors (in $\mathbb{R}^{k(n+2)(n+1) / 2}$ ) of $k$-CPs. This is discussed in section 4 .

\section{Minimum cost $k-\mathrm{CPs}$}

We describe a procedure for the minimum cost $k$-CP similar to the one suggested by Dell'Amico, Fischetti and Toth [3] for the multiple depot vehicle scheduling problem.

From $G_{k}$ a graph $G$ is constructed in which the nodes are settled by stages. In stage 0 there is a unique node which is the $k$-tuple $(0,0, \ldots, 0)$. The nodes of stage $j=1,2, \ldots, n$ are all the different $k$-tuples $\left(s_{1}^{j}, s_{2}^{j}, \ldots, s_{k}^{j}\right)$ obtained from every node $\left(s_{1}^{j-1}, s_{2}^{j-1}, \ldots, s_{k}^{j-1}\right)$, of stage $j-1$, by replacing exactly one of its components by $j$. Thus, the nodes of stage 
$j$ are all the $k$-tuples consisting of integers $0,1, . ., j$ where $j$ occurs exactly once, and in which only 0 may occur more than once. The number of nodes of stage $j$ is $O\left(k j^{k-1}\right)$.

There is an arc from the $j-1$ stage node $\left(s_{1}^{j-1}, \ldots, s_{l}^{j-1}, \ldots, s_{k}^{j-1}\right)$ to node $\left(s_{1}^{j-1}, \ldots\right.$, $\left.s_{l-1}^{j-1}, j, s_{l+1}^{j-1}, \ldots, s_{k}^{j-1}\right)$, of stage $j$. Define the length of this arc of $G$ as equal to the cost of the $l$ colour arc $\left(s_{l}^{j-1}, j\right)$ of $G_{k}$.

Graph $G$ also has the $k$-tuple $(n+1, n+1, \ldots, n+1)$ as a node. There is an arc from each node $\left(s_{1}^{n}, s_{2}^{n}, \ldots, s_{k}^{n}\right)$, of stage $n$, to $(n+1, n+1, \ldots, n+1)$. The length of this arc is defined as the sum of the costs of the $\operatorname{arcs}\left(s_{l}^{n}, n+1\right)$ of $G_{k}$ in each of the $k$ colours. No further nodes and arcs exist.

Paths in $G$ from $(0,0, \ldots, 0)$ to $(n+1, n+1, \ldots, n+1)$ and $k$-CPs of $G_{k}$ are in a 1 to 1 correspondence. Thus, obtaining a minimum cost $k$-CP in $G_{k}$ amounts to look for a shortest path from $(0, \ldots, 0)$ to $(n+1, \ldots, n+1)$ in graph $G$, which has $O\left(k n^{k}\right)$ nodes. This gives an $O\left(k^{2} n^{2 k}\right)$ time algorithm, hence polynomial for any fixed $k$.

We illustrate the construction of $G$ from the graph $G_{2}$ of Figure 1 where $n=3$, colour 1 is blue, colour 2 is red, and the costs of the arcs are displayed in the figure. The missing arcs have costs equal to $+\infty$.

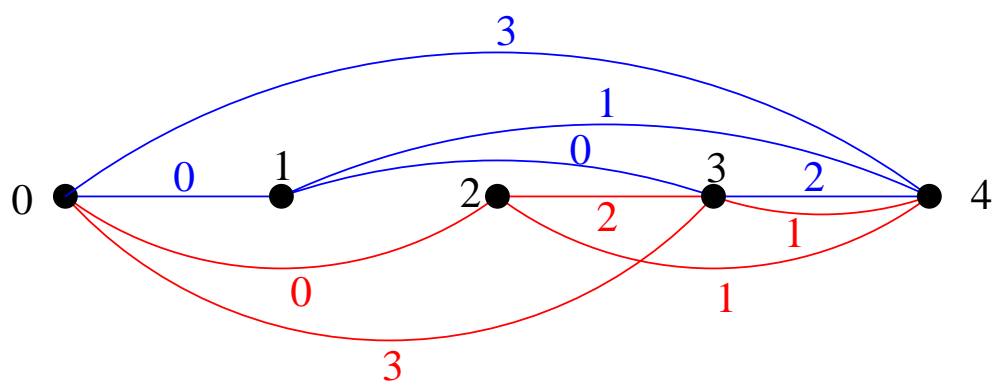

Figure 1: The graph $G_{2}$

The resulting graph $G$ and the lengths of its arcs are shown in Figure 2. The path $((0,0),(1,0),(1,2),(3,2),(4,4))$, of length 3 , is the shortest path from $(0,0)$ to $(4,4)$. The corresponding 2 -CP consists of the blue path $(0,1,3,4)$ and the red path $(0,2,4)$.

To show that finding $k$-CPs of minimum cost is NP-hard, consider the $k$-CP decision problem which asks whether a spanning subgraph $G_{k}^{\prime}$ of $G_{k}$ includes a $k$-CP. We use the NP-completeness of the satisfiability problem (SAT) to prove the following lemma. (We follow the terminology from the description of SAT given in Garey and Johnson [4].)

Theorem 1 The $k$-CP decision problem is NP-complete.

Proof. The problem is clearly in NP. We transform SAT in to the $k$-CP decision problem. Consider an instance of SAT, i.e., a set $X=\left\{x_{1}, \ldots, x_{m}\right\}$ of boolean variables and a collection $C=\left\{c_{1}, \ldots, c_{t}\right\}$ of clauses over $X$. The corresponding instance of the $k$-CP decision problem is $k:=2 m$ and graph $G_{k}^{\prime}$ defined as follows. The node set of $G_{k}^{\prime}$ is 


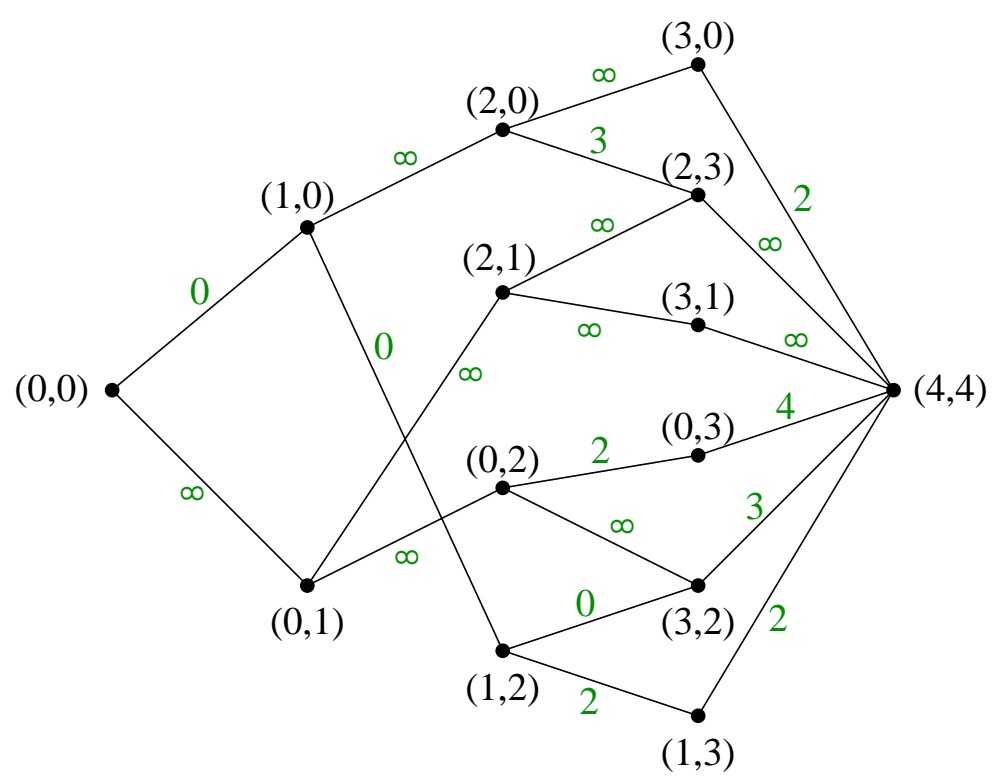

Figure 2: The graph $G$

$\{0,1, \ldots, n, n+1\}$, with $n=m+t$. The node $j=1, \ldots, m$ corresponds to variable $x_{j}$. The node $j=m+1, \ldots, m+t$ corresponds to clause $c_{j-m}$. There are two arcs from 0 to each node $j=1, \ldots, m$. One has colour $j$ and the other the colour $m+j$. For $j=1, \ldots, m$, if $c_{j_{1}}, c_{j_{2}}, \ldots, c_{j_{s}}$, with $j_{1}<j_{2}<\cdots<j_{s}$, are the clauses in which literal $x_{j}$ $\left(\bar{x}_{j}\right)$ occurs, $G_{k}^{\prime}$ includes every arc from $m+i$ to $m+l, i<l=j, j_{1}, \ldots, j_{s}, n+1$, with colour $j(m+j)$. Finally, $G_{k}^{\prime}$ also has $2 m$ different colour arcs connecting 0 to $n+1$. No further arcs exist.

Given any $k$-CP of $G_{k}^{\prime}$ the following procedure defines a satisfying truth assignment $T$ on $X$. Note that node $j=1, \ldots, m$ can only be covered by either the $j$ colour arc $(0, j)$, or the $m+j$ colour arc $(0, j)$. If colour $j$ is used to cover node $j$, then define $T\left(x_{j}\right):=$ true. If colour $m+j$ is used, define $T\left(x_{j}\right):=$ false. Since any $k$-CP covers all nodes $j=1, \ldots, m$, $T$ is a well defined truth assignment.

If node $j$ is reached with colour $j$, the $k$-CP includes a colour $j$ path from $j$ to $n+1$ in which all interior nodes correspond to clauses where literal $x_{j}$ occurs, and hence every such clause is satisfied by $T\left(x_{j}\right)=$ true. If node $j$ is reached with colour $m+j$, the $k$-CP includes a colour $m+j$ path from $j$ to $n+1$ in which all interior nodes correspond to clauses where literal $\bar{x}_{j}$ occurs, and every such clause is satisfied by $T\left(x_{j}\right)=$ false. Since any $k$-CP covers all nodes corresponding to clauses, all are satisfied showing that $T$ is a satisfying truth assignment.

Given any satisfying truth assignment $T$ the following procedure finds a pair of paths in $G_{k}^{\prime}$, of colours $j$ and $m+j$, for $j=1, \ldots, m$.

If $T\left(x_{j}\right)=$ true $\left(T\left(x_{j}\right)=\right.$ false $)$ the colour $j(m+j)$ path from 0 to $n+1$ includes node $j$ and every node corresponding to clauses where literal $x_{j}\left(\bar{x}_{j}\right)$ occurs and which is not yet in any of the previously defined paths $1, \ldots, j-1$. The $m+j(j)$ colour path from 
0 to $n+1$ consists of the single arc $(0, n+1)$. Since $T$ is a satisfying truth assignment, these $2 m$ paths define a $k$-CP.

We illustrate the above proof using the instance of SAT consisting of the set $X=$ $\left\{x_{1}, x_{3}, x_{3}\right\}$ of variables, and clauses $c_{1}=\left(x_{1} x_{2} \bar{x}_{3}\right), c_{2}=\left(x_{1} \bar{x}_{2} x_{3}\right), c_{3}=\left(\bar{x}_{1} x_{2}\right)$. The corresponding graph is $G_{6}^{\prime}$ of Figure 3 , where the 6 colours are continuous and hatched blue, red and green.

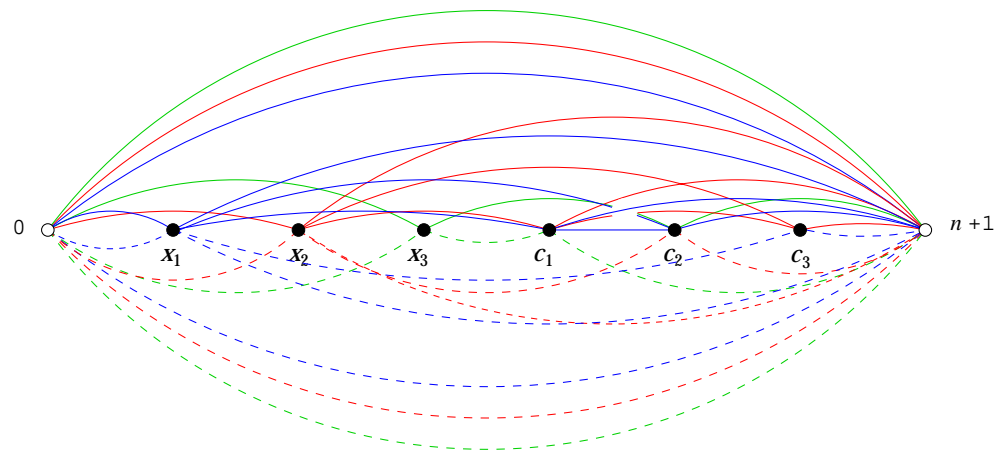

Figure 3: The graph $G_{6}^{\prime}$

\section{The 2-CP polytope}

In this section we make a polyhedral study of 2-CPs. For simplicity colours 1 and 2 will be referred to as blue and red respectively.

Let $x^{b}, x^{r} \in \mathbb{R}^{(n+2)(n+1) / 2}$ denote, respectively, the incidence vectors of a set of blue and a set of red arcs of $G_{2}$. The incidence vectors of 2-CPs are the pairs $\left(x^{b}, x^{r}\right)$ which satisfy the following equations:

$$
\begin{gathered}
\sum_{i=0}^{j-1}\left(x_{i j}^{b}+x_{i j}^{r}\right)=1, \quad j=1, \ldots, n \\
\sum_{i=0}^{j-1} x_{i j}^{b}-\sum_{i=j+1}^{n+1} x_{j i}^{b}=0, \quad j=1, \ldots, n \\
\sum_{i=1}^{n+1} x_{0 i}^{b}=1 \\
\sum_{i=0}^{j-1} x_{i j}^{r}-\sum_{i=j+1}^{n+1} x_{j i}^{r}=0, \quad j=1, \ldots, n
\end{gathered}
$$




$$
\sum_{i=1}^{n+1} x_{0 i}^{r}=1
$$

Equations (2) and (4) are the usual flow conservation constraints. Thus, (2) and (3) ((4) and (5)) define $x^{b}\left(x^{r}\right)$ to be a blue (red) path from 0 to $n+1$. Equalities (1) ensure that each node of $N=\{1,2, \ldots, n\}$ is included either in the blue or in the red path.

Note that given any $x^{b}$ fulfilling (2) and (3), equations (1),(4) and (5) produce a unique vector $x^{r}$, which we call the red counterpart of $x^{b}$, and denote by $R\left(x^{b}\right)$.

Let $\mathcal{C}_{2-\mathrm{CP}} \subseteq \mathbb{R}^{(n+2)(n+1)}$ be the set of incidence vectors of 2-CPs, and consider $L \subseteq$ $\mathcal{C}_{2-\mathrm{CP}}$ consisting of all pairs $\left(x^{b}, R\left(x^{b}\right)\right)$, where $x^{b}$ is the incidence vector of a blue path from 0 to $n+1$ which

(i) includes no more than two nodes from $N$;

(ii) includes one path of at least three consecutive nodes from $N$ and no other arc with both nodes in $N$, i.e., $(0, i, i+1, \ldots, i+k, n+1)$ with $k \geq 2$.

It is easy to verify the following result.

Lemma 2 The incidence vector of every path from 0 to $n+1$ is an affine combination of incidence vectors of paths of type (i).

Proof. Let $x$ be the incidence vector of path $\left(0, i_{1}, i_{2}, \ldots, i_{k}, n+1\right)$, with at least three nodes in $N$. Then $x=\sum_{l=1}^{k-1} y_{l}-\sum_{l=2}^{k-1} z_{l}$, in which $y_{l}$ is the incidence vector of path $\left(0, i_{l}, i_{l+1}, n+1\right)$ and $z_{l}$ the incidence vector of path $\left(0, i_{l}, n+1\right)$. Moreover, the above combination is unique, since the set of incidence vectors of paths of type (i) is clearly linearly independent.

Yet there are 2-CPs whose incidence vectors are not linear combinations of the incidence vectors of 2 -CPs of type (i). Indeed we have the following.

Lemma 3 The set $L$ is linearly independent.

Proof. The set of incidence vectors of 2-CPs of type (i) is obviously linearly independent. The following observation completes the proof: Every 2-CP of type (ii) with the blue path $(0, i, i+1, \ldots, i+k, n+1), k \geq 2$, is the unique member of $L$ which includes the red arc $(i-1, i+k+1)$.

Lemma 4 Every $\left(x^{b}, R\left(x^{b}\right)\right) \in \mathcal{C}_{2-\mathrm{CP}}$ is an affine combination of $L$.

Proof. If the blue path defined by $x^{b}$ has no nodes in $N$, i.e., consists of the blue arc $(0, n+1)$, then $\left(x^{b}, R\left(x^{b}\right)\right) \in L$ and the proof is complete. Hence we will consider that the path defined by $x^{b}$ includes at least one node from $N$.

Every such path may be viewed as a sequence of $k \geq 1$ paths, each consisting of consecutive nodes from $N$, connected by arcs of nonconsecutive nodes. If $P(i, j)$, with $i \leq j$, denotes the path $(i, i+1, \ldots, j)$ of consecutive nodes of $N$, then $x^{b}$ is the incidence 
vector of the blue path $\left(0, P\left(i_{1}, j_{1}\right), P\left(i_{2}, j_{2}\right), \ldots, P\left(i_{k}, j_{k}\right), n+1\right)$, with $i_{l}>j_{l-1}+1$, for $l=2, \ldots, k$. Assuming $P(i, j)=\emptyset$ in case $i>j$, the red counterpart of $x^{b}$ is the incidence vector of the red path $\left(0, P\left(1, i_{1}-1\right), P\left(j_{1}+1, i_{2}-1\right), \ldots, P\left(j_{k}+1, n\right), n+1\right)$.

To prove $\left(x^{b}, R\left(x^{b}\right)\right)$ is an affine combination of elements of $L$ we use induction on $k$. For $k=1$ the result obviously holds as $\left(x^{b}, R\left(x^{b}\right)\right) \in L$.

For $k>1$ let $\bar{x}^{b}$ be the incidence vector of the blue path consisting of the $k-1$ first $P\left(i_{l}, j_{l}\right)$ of $x^{b}$, i.e., $\left(0, P\left(i_{1}, j_{1}\right), P\left(i_{2}, j_{2}\right), \ldots, P\left(i_{k-1}, j_{k-1}\right), n+1\right)$. Consider also $y^{b}, z^{b}$, $w^{b}$ and $v^{b}$ the incidence vectors of the blue paths $\left(0, P\left(i_{k}, j_{k}\right), n+1\right),\left(0, j_{k-1}, i_{k}, n+1\right)$, $\left(0, j_{k-1}, n+1\right)$ and $\left(0, i_{k}, n+1\right)$, respectively.

It is now easy to check that we have both $x^{b}=\bar{x}^{b}+y^{b}+z^{b}-w^{b}-v^{b}$ and $R\left(x^{b}\right)=$ $R\left(\bar{x}^{b}\right)+R\left(y^{b}\right)+R\left(z^{b}\right)-R\left(w^{b}\right)-R\left(v^{b}\right)$. Induction shows that $\left(x^{b}, R\left(x^{b}\right)\right)$ is an affine combination of elements of $L$.

Theorem 5 The dimension of the convex hull of $\mathcal{C}_{2-\mathrm{CP}}$ is $n^{2}-n+1$.

Proof. This follows immediately from $|L|=n^{2}-n+2$ and Lemmas 3 and 4 .

We will now establish a complete system of equalities for the convex hull of $\mathcal{C}_{2-\mathrm{CP}}$. Theorem 5 ensures that at least $4 n+1$ equations are needed. System (1)-(5), consisting of $3 n+2$ equations, is therefore not sufficient.

Consider equations

$$
\sum_{i=0}^{j-1}\left(x_{i j}^{b}+x_{i j+1}^{b}\right)+x_{j j+1}^{r}=1 \quad j=1, \ldots, n-1,
$$

which, when $x^{b}$ and $x^{r}$ are $0 / 1$ vectors, are implied from (1)-(5). Each equation states, in one hand, that if red arc $(j, j+1)$ is included in the path defined by $x^{r}$, nodes $j$ and $j+1$ are not used by the blue path defined $x^{b}$. On the other hand, it also states that if red $\operatorname{arc}(j, j+1)$ is not included in the path defined by $x^{r}$, then $j$ or $j+1$ is used by the blue path defined by $x^{b}$, and that if both $j$ and $j+1$ are used, the path also includes the blue $\operatorname{arc}(j, j+1)$.

When $x^{b}$ and $x^{r}$ are not constrained to $0 / 1$ values, equations (6) do not follow from (1)-(5). Let $A$ be the matrix of the coefficients of the $4 n+1$ equations (1)-(6).

Lemma 6 Matrix $A$ is of full row rank.

Proof. Each arc $(j, n+1), j=0, \ldots, n$, of each of the two colours interferes in exactly one of the flow constraints (2)-(5). None of these arcs appears in equations (1), and each equation uses exactly one red $\operatorname{arc}(0, j), j=1, \ldots, n$. This shows that the set of the $3 n+2$ rows of $A$ corresponding to equations (1)-(5) is linearly independent.

Each red arc $(j, j+1), j=1, \ldots, n-1$ figures in exactly one equality (6). None of these equations involves any of the previous arcs.

Theorem 5 and Lemma 6 ensure that (1)-(6) is a complete minimal system of equations for the convex hull of $\mathcal{C}_{2-\text { CP. }}$. In what follows we prove that $x^{b}, x^{r} \geq 0$ are the only inequalities which are needed to obtain a full description of the convex hull of $\mathcal{C}_{2-\mathrm{CP}}$. 
We will show this by giving an algorithm which decomposes any member of $\mathcal{P}_{2-\mathrm{CP}}=$ $\left\{\left(x^{b}, x^{r}\right) \geq 0:(1)-(6)\right\}$ into a convex combination of elements in $\mathcal{C}_{2-\mathrm{CP}}$.

More specifically, for $0<\lambda \leq 1$ consider $y=\lambda x$ with $x \in \mathcal{P}_{2-\text { CP }}$, i.e., $y \geq 0$ satisfies $\left(1_{\lambda}\right)-\left(6_{\lambda}\right)$, which are equations (1)-(6) with the right hand sides multiplied by $\lambda$. Let $G_{y}$ be the graph obtained from $G_{2}$ removing every blue (red) arc $(i, j)$ for which $y_{i j}^{b}=0$ $\left(y_{i j}^{r}=0\right)$. We will exhibit $\bar{x} \in \mathcal{C}_{2-\mathrm{CP}}$ and $0<\alpha \leq \lambda$, such that the blue and red paths defined by $\bar{x}$ are subgraphs of $G_{y}$, and $G_{y-\alpha \bar{x}}$ is a proper subgraph of $G_{y}$. Thus, starting from an arbitrary $y \in \mathcal{P}_{2-\text { CP }}$, i.e., from $\lambda=1$, in each step $k$ we determine a 2 -CP $\bar{x}(k)$ and a value $\alpha(k)$ and define $y:=y-\alpha(k) \bar{x}(k) \geq 0$. The procedure will eventually stop, since in each step at least one arc is removed from the current $G_{y}$, defining the initial $y$ as the convex combination of incidence vectors of 2-CPs: $\sum_{k} \alpha(k) \bar{x}(k)$.

We first give two technical lemmas.

Lemma 7 Let $y$ and $G_{y}$ be defined as above, and $1 \leq u<v \leq n$. Then $\sum_{k<u} \sum_{l>v} y_{k l}^{b} \leq$ $y_{j j+1}^{r}$, for $j=u, \ldots, v-1$. Thus, the existence of any blue arc $(k, l)$ in $G_{y}$, with $k<u$ and $l>v$, implies the red path $(u, u+1, \ldots, v)$ to be also in $G_{y}$.

Proof. Equations $\left(2_{\lambda}\right)$ and $\left(3_{\lambda}\right)$ ensure $\sum_{i<j} \sum_{l \geq j} y_{i l}^{b}=\lambda$, for all $j \in N$. If $\alpha=$ $\sum_{k<u} \sum_{l>v} y_{k l}^{b}$ then $\sum_{i<j}\left(y_{i j}^{b}+y_{i j+1}^{b}\right) \leq \lambda-\alpha$, for $j=u, \ldots, v-1$. Using $\left(6_{\lambda}\right), \lambda=$ $\sum_{i<j}\left(y_{i j}^{b}+y_{i j+1}^{b}\right)+y_{j j+1}^{r} \leq \lambda-\alpha+y_{j j+1}^{r}$, and therefore $y_{j j+1}^{r} \geq \alpha$.

Lemma 8 Let $y$ and $G_{y}$ be defined as above, and $1 \leq j \leq n$. Then $\sum_{i<j} y_{i j+1}^{b}=$ $\sum_{l>j+1} y_{j l}^{r}$. Thus, the existence of any blue arc $(i, j+1)$ in $G_{y}$, with $i<j$, implies that at least one of the red arcs $(j, l), l>j+1$, is also in $G_{y}$.

Proof. Equations $\left(1_{\lambda}\right)$ and $\left(4_{\lambda}\right)$ imply $\sum_{i<j} y_{i j}^{b}+\sum_{l>j} y_{j l}^{r}=\lambda$. From $\left(6_{\lambda}\right)$ and the previous equality we have $\sum_{i<j}\left(y_{i j}^{b}+y_{i j+1}^{b}\right)+y_{j j+1}^{r}=\sum_{i<j} y_{i j}^{b}+\sum_{l>j} y_{j l}^{r}$, and therefore $\sum_{i<j} y_{i j+1}^{b}=\sum_{l>j+1} y_{j l}^{r}$.

Remark 9 Lemmas 7 and 8 also apply when switching the blue and red colours.

Proof. Note that instead of equations (6) we could have used

$$
\sum_{i=0}^{j-1}\left(x_{i j}^{r}+x_{i j+1}^{r}\right)+x_{j j+1}^{b}=1, \quad j=1, \ldots, n-1 .
$$

Indeed system (1),(6') is equivalent to $(1),(6)$.

We are now on conditions to prove the main result.

Theorem $10 \mathcal{P}_{2-\mathrm{CP}}=\left\{\left(x^{b}, x^{r}\right) \geq 0:(1)-(6)\right\}$ is the convex hull of $\mathcal{C}_{2-\mathrm{CP}}$.

Proof. Let $y$ and graph $G_{y}$ be defined as above. We construct a 2-CP $\bar{X}$ using arcs of $G_{y}$. Take any arc $(0, j)$ with $j>1$ and define $\bar{X}:=\{(0, j)\}$. One such arc has to exist since otherwise $y_{01}^{b}+y_{01}^{r}=2 \lambda$ contradicting $\left(1_{\lambda}\right)$. Suppose, without loss of generality (given $9)$, that arc $(0, j)$ is blue. Lemma 7 ensures the existence of the red path $(1,2, \ldots, j-1)$ 
. Equation $\left(5_{\lambda}\right)$ and flow conservation constraint $\left(4_{\lambda}\right)$ on node 1 implies that the red arc $(0,1)$ also exists. Now, Lemma 8 shows there is a red $\operatorname{arc}(j-1, l)$, with $l>j$. Add to $\bar{X}$ the red path $(0,1, \ldots, j-1, l)$. Lemma 8 and Remark 9. Put the blue path $(j, \ldots, l-1, k)$ in $\bar{X}$.

Continue proceeding this way until node $n+1$ is reached by one of the two paths. This will certainly occur when using Lemma 8 (if needed together with Remark 9) and some arc $(t, n+1)$, with $t<n$, is added to $\bar{X}$. Suppose $(t, n+1)$ is a blue arc. Surely some red arc $(s, t+1)$, with $s<t$, has already been added to $\bar{X}$. If $t+1<n$, Lemma 7 ensures that red path $(t+1, t+2, \ldots, n)$ is in $G_{y}$. Equation $\left(5_{\lambda}\right)$ and flow conservation constraint $\left(4_{\lambda}\right)$ on node $n$ implies that red arc $(n, n+1)$ also exists. Adding the red path $(t+1, \ldots, n, n+1)$ to $\bar{X}$ turns this set into a 2 -CP.

Let $\bar{x}$ be the incidence vector of $\bar{X}$ and define $\alpha$ to be the minimum component of $y$ among the arcs of $\bar{X}$. Recall $y=\lambda x$ with $x \in \mathcal{P}_{2-\mathrm{CP}}$ and set $y:=y-\alpha \bar{x} \geq 0$. Note that now $y=(\lambda-\alpha) x$ and the new $G_{y}$ is a proper subgraph of the previous one.

This shows how to turn an arbitrary $y=x \in \mathcal{P}_{2-\mathrm{CP}}$ (i.e., $\lambda=1$ ) into a convex combination of 2-CPs $\bar{x}$ with coefficients $\alpha$.

\section{The $k$-CP polytope}

The system of equations (1)-(5) straightforwardly extends to $k$-CPs. If $x^{l} \in \mathbb{R}^{(n+2)(n+1) / 2}$ denotes the incidence vector of the set of arcs of colour $l$, the incidence vectors of $k$-CPs are the $(k(n+2)(n+1) / 2)$-tuples $\left(x^{l}, l=1, \ldots, k\right)$ which satisfy the following equations:

$$
\begin{gathered}
\sum_{l=1}^{k} \sum_{i=0}^{j-1} x_{i j}^{l}=1, \quad j=1, \ldots, n \\
\sum_{i=0}^{j-1} x_{i j}^{l}-\sum_{i=j+1}^{n+1} x_{j i}^{l}=0, \quad j=1, \ldots, n ; \quad l=1, \ldots, k \\
\sum_{i=1}^{n+1} x_{0 i}^{l}=1, \quad l=1, \ldots, k
\end{gathered}
$$

Let $B$ be the matrix of the coefficients of the $(k+1) n+k$ equations (7)-(9).

Lemma 11 Matrix $B$ is of full row rank.

Proof. We extend the first part of the proof of Lemma 6 to the case $k>2$.

Each arc $(j, n+1), j=0, \ldots, n$, of each of the $k$ colours interferes in exactly one of the flow constraints (8)-(9). None of these arcs appears in equations (7), and each equation uses exactly one of the colour $k$ arcs $(0, j), j=1, \ldots, n$. This shows that the set of the $(k+1) n+k$ rows of $B$ is linearly independent.

Denote by $\mathcal{C}_{\mathrm{k}-\mathrm{CP}} \subseteq \mathbb{R}^{k(n+2)(n+1) / 2}$ the set of incidence vectors of $k$-CPs and let $\mathcal{P}_{\mathrm{k}-\mathrm{CP}}$ be the convex hull of $\mathcal{C}_{\mathrm{k}-\mathrm{CP}}$. 
Unlike the case when $k=2$, where to derive an equality set for $\mathcal{P}_{\mathrm{k}-\mathrm{CP}}$ we had to add to (7)-(9) some extra equations, we will see that for $k>2$ equalities (7)-(9) are enough. We prove this by exhibiting an affinely independent set $S_{j}^{l}$ of $\frac{1}{2}\left(k n^{2}+k n-2 n\right)+1$ members of $\mathcal{C}_{\mathrm{k}-\mathrm{CP}}$. Since $\left|S_{j}^{l}\right|-1$ is precisely the dimension of the ground set of $\mathcal{P}_{\mathrm{k}-\mathrm{CP}}$ minus the number of equations (7)-(9), it follows from Lemma 11 that $\operatorname{dim} \mathcal{P}_{\mathrm{k}-\mathrm{CP}}=\frac{1}{2}\left(k n^{2}+k n-2 n\right)$ and that (7)-(9) is a minimal equality set for $\mathcal{P}_{\mathrm{k}-\mathrm{CP}}$.

Let $l$ be any of the $k$ colours and consider

(iii) for some colour $t \neq l$, the 2-CPs that use colour $l$ and include a $t$ colour path of type (i) or type (ii) from 0 to $n+1$,

(iv) for each colour $p \neq l$, the the -CPs that use colour $l$ and include a $p$ colour path of type (i) from 0 to $n+1$, different from $(0, n+1)$.

There are $|L|=n^{2}-n+22$-CPs of type (iii) and $(k-2)(n+n(n-1) / 2)$ of type (iv).

Consider also, for some colour $p \neq l, t$ and for $i=1, \ldots, n$, the 2-CPs that

(v) use colour $t$ and include the $p$ colour path $(0, i, n+1)$.

By adding to any 2-CP above the $k-2$ arcs $(0, n+1)$ of each of the remaining colours, a $k$-CP is obtained. Let $s_{i}, i=1, \ldots, n$, be the incidence vector of the $k$-CP resulting from the 2-CP of type (v) and, for any given $j=1, \ldots, n$, define $S_{j}^{l}$ to be the set of the incidence vectors of the $k$-CPs obtained from every 2-CP (iii), (iv) or (v), different from $s_{j}$.

Lemma 12 The set $S_{j}^{l} \subseteq \mathcal{C}_{\mathrm{k}-\mathrm{CP}}$ is linearly independent.

Proof. Let $C$ be the set of $k$-CPs whose incidence vectors are in $S_{j}^{l}$. We first identify some $k$-CPs in $C$ which, for the purpose of proving independence of $S_{j}^{l}$, can be removed from consideration.

If $p \neq l, t$ and for $i=1, \ldots, n-1$, there is a unique $k$-CP in $C$ with the $p$ colour arc $(i, i+1)$. This is the $k$-CP obtained from the 2-CP of type (iv) with the $p$ colour path of type (i) $(0, i, i+1, n+1)$, and the $l$ colour arc $(i-1, i+2)$. Thus, if we delete from $C$ every such $k$-CP, $S_{j}^{l}$ is linearly independent iff the set of incidence vectors of the remaining $k$-CPs of $C$ is also independent. We proceed with this reduced set $C$.

Any $l$ colour arc $(i, i+r) \neq(0, n+1)$, with $r>2$, appears in a unique member of the current set $C$. This is the $k$-CP obtained from the 2-CP of type (iii) which includes the $t$ colour path $(0, i+1, i+2, \ldots, i+r-1, n+1)$. All these $k$-CPs can also be removed from C.

Note that in the new set $C$ the $k$-CPs which use any $t$ colour arc $(i, i+1)$, with $i=1, \ldots, n-1$, are those whose incidence vectors are $s_{1}, s_{2}, \ldots, s_{n}\left(\neq s_{j}\right)$ and the one resulting from the 2 -CP of type (iii) with the $t$ colour path $(0,1, \ldots, n+1)$, whose incidence vector we denote by $s_{0}$. Also note that the $l$ colour arc $(0, n+1)$ appears in all these $k$-CPs, and in no other $k$-CP of $C$. For $r=0,1, \ldots, n$, let $p_{r}$ be the restriction of $s_{r}$ to the indices which correspond to the $l$ colour arc $(0, n+1)$ and $t$ colour $\operatorname{arcs}(i, i+1)$, 
$i=1, \ldots, n-1$. Since the set of incidence vectors of $k$-CPs of $C$ different from $s_{r}$ is clearly independent, it follows from the previous remarks that the proof can be completed by showing that $\cup_{r=0 ; r \neq j}^{n}\left\{p_{r}\right\}$ is linearly independent. Hence, consider the $n \times n$ matrix with column $r$ equal to $p_{r}, r=0, \ldots, n ; r \neq j$. This matrix can be viewed as obtained by deleting the column corresponding to $s_{j}$ from the $n \times(n+1)$ matrix

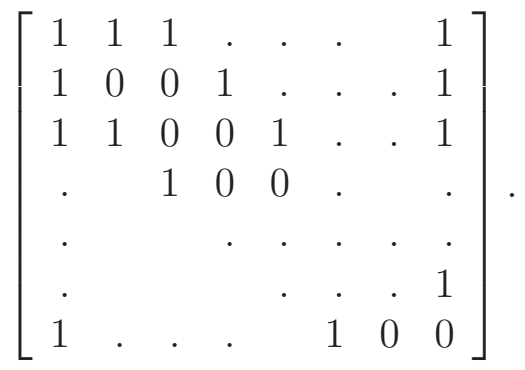

It is straightforward to verify that every $n \times n$ submatrix of such a matrix obtained by deleting any column, different from the first one, is nonsingular.

We are now on conditions to establish, for $k>2$, the dimension of the convex hull of the set of incidence vectors of $k$-CPs.

Theorem 13 If $k>2$, $\operatorname{dim} \mathcal{P}_{\mathrm{k}-\mathrm{CP}}=\frac{1}{2}\left(k n^{2}+k n-2 n\right)$.

Proof. Lemmas 11 and 12 imply that $\left|S_{j}^{l}\right|-1 \leq \operatorname{dim} \mathcal{P}_{\mathrm{k}-\mathrm{CP}} \leq m-b$, where $m=\frac{k(n+2)(n+1)}{2}$ is the dimension of the ground set of $\mathcal{P}_{\mathrm{k}-\mathrm{CP}}$ and $b=(k+1) n+k$ is the number of equations (7)-(9). Since $\left|S_{j}^{l}\right|-1=m-b=\frac{1}{2}\left(k n^{2}+k n-2 n\right)$, the result follows.

Theorem 13 allows to conclude that, if $k>2$, every equation satisfied by all elements of $\mathcal{P}_{\mathrm{k}-\mathrm{CP}}$ is implied by equalities (7)-(9). Extending equations (6) for $k>2$ the following valid inequalities were obtained

$$
\sum_{i=0}^{j-1}\left(x_{i j}^{l}+x_{i j+1}^{l}\right)+\sum_{p=1 ; p \neq l}^{k} x_{j j+1}^{p} \leq 1 \quad j=1, \ldots, n-1 ; \quad l=1, \ldots, k
$$

Note that for each inequality we can find incidence vectors of $k$-CPs $\left(s_{j}\right.$ and $s_{j+1}$, for example) for which the left hand side is strictly less than 1 .

We finish with a result regarding the inequalities (10).

Theorem 14 For $k>2$, each inequality (10) determines a facet of $\mathcal{P}_{\mathrm{k}-\mathrm{CP}}$.

Proof. We show that every element of $S_{j}^{l} \backslash\left\{s_{j+1}\right\}$ satisfies the corresponding inequality (10) with equality.

Equations (6) hold for the incidence vector of every 2-CP. Therefore, the incidence vector of a $k$-CP obtained adding to a 2 -CP the $k-2 \operatorname{arcs}(0, n+1)$ of each of the remaining colours, satisfy with equality every inequality (10) in which $l$ is any of the 2 colours of the 2-CP. Thus, the incidence vectors of the $k$-CPs obtained from 2 -CPs of 
types (iii) and (iv) satisfy (10) with equality. The same happens with every $s_{i} \in S_{j}^{l}$, except $s_{j+1}$, since the corresponding $k$-CP uses the $t \neq l$ colour arc $(j, j+1)$.

The result now follows from Lemma $12,\left|S_{j}^{l} \backslash\left\{s_{j+1}\right\}\right|=\operatorname{dim} \mathcal{P}_{\mathrm{k}-\mathrm{CP}}$, and from the arbitrary choice of $l=1, \ldots, k$ and $j=1, \ldots, n$ in defining $S_{j}^{l}$.

\section{References}

[1] J. Bang-Jensen and G. Gutin, Digraphs: theory, algorithms and applications, London, Springer 2001.

[2] C. Berge, Path partitions in directed graphs, Annals of Discrete Mathematics 17 (1983), 59-63.

[3] M. Dell'Amico, M. Fischetti and P. Toth, Heuristic algorithms for the multiple depot vehicle scheduling problem, Management Science 39 (1993), 115-125.

[4] M. R. Garey and D. S. Johnson, Computers and intractability: a guide to the theory of NP completeness, San Franscico, W. H. Freeman \& Company 1979.

[5] A. Schrijver, Polyhedral combinatorics, in R.L. Graham, M. Grötschel, L. Lovász, ed., Handbook of Combinatorics, Vol. II, Elsevier, 1649-1704, 1995. 